\title{
Policing and group cohesion when resources vary
}

\author{
STEVEN A. FRANK \\ Department of Ecology and Evolutionary Biology, University of California at Irvine \\ (Received 5 January 1996; initial acceptance 23 March 1996; \\ final acceptance 26 April 1996; MS. number: A7490)
}

\begin{abstract}
The transition from competing individuals to cooperative groups has occurred several times in evolutionary history. The puzzle is why selfish individuals did not subvert cohesive group behaviour by taking resources without contributing to the group's overall success. Kin selection and reciprocal altruism are the two standard explanations for group cohesion. But many groups have evolved into cooperative units when relatedness was low and opportunities were limited for the strategic alliances required for reciprocity. A new theory was recently proposed in which individuals invest some of their resources into repressing competition between group members. Such policing increases the fair distribution of resources in the group and enhances group cohesion. The surprising aspect of this theory is that low relatedness is more conducive to the spread of policing traits than is high relatedness. Here a new explanation is developed of the biological processes that favour policing. The model is then extended in two ways. First, more realism is added to the theory by accounting for the full range of costs and benefits associated with competitive and cooperative traits within groups. Second, another surprising result is introduced about cooperative evolution. Small variations in individual vigour or resources can lead to large variations in individual contributions to policing the group. Stronger individuals often invest all of their excess resources into policing, but weaker individuals do not contribute to group cohesion.

(C) 1996 The Association for the Study of Animal Behaviour
\end{abstract}

An important puzzle in biology is how groups of independent units evolve to form a cohesive functional unit (Maynard Smith \& Szathmáry 1995). The problem is the 'tragedy of the commons', in which competition for limited resources favours rapacious individuals over prudent ones. This competition between neighbours reduces the sustainable yield of the group, lowering the fitness of all members.

There are two standard explanations for group cooperation. Kin selection favours self-restraint and prudent behaviour between genetically similar neighbours (Hamilton 1964). Strategic alliance favours cooperation with neighbours who reciprocate, as in the famous Tit-for-Tat strategy of game theory (Axelrod 1984).

These two ideas, although fundamental to many important problems, are not sufficient to explain several aspects of cooperative evolution. For example, the puzzle of early cellular life is

Correspondence: S. A. Frank, Department of Ecology and Evolutionary Biology, University of California, Irvine, CA 92697-2525, U.S.A. (email: safrank@uci.edu). how different kinds (species) of replicators evolved into a functional unit. Kin selection cannot be the sole factor, because the key interactions are between different species. Strategic alliance models of game theory also fail because they usually require recognition of individuals and memory of past interactions. Even in organisms with the potential for memory and complex strategy, the form of mixing and competition often precludes game theory solutions.

I recently proposed a theory to explain how competition between lower-level units is suppressed in the formation of higher-level evolutionary units (Frank 1995; extending earlier work by Alexander 1987; Ratnieks \& Visscher 1989; Wilson \& Sober 1994). The idea is that individuals may invest some of their resources in repressing competition among group members. Such policing increased the fair distribution of resources in the group, enhances group efficiency and raises the average fitness of group members.

The surprising aspect of my new theoretical work is how readily policing traits increase in frequency when relatedness among group 
members is low. Indeed, low relatedness is more conducive to the spread of policing traits than is high relatedness.

Here I show another surprising result in cooperative evolution. Small variations in individual vigour or resources can lead to large variations in individual contributions to policing the group. Stronger individuals often invest all of their excess resources into policing, but weaker individuals do not contribute to group cohesion.

\section{CLARIFICATION OF THE ORIGINAL MODEL}

My previous work on this subject was published in a brief note (Frank 1995). Subsequent correspondence showed some confusion about the policing theory and, more generally, about kin selection in group-structured models. In this section I provide new analyses to explain clearly why low relatedness is conducive to group policing.

\section{Review}

The model begins with the costs and benefits of group competition

$$
w_{i j}=\left(z_{i j} / z_{i}\right)\left(1-z_{i}\right)
$$

where $w_{i j}$ and $z_{i j}$ are the fitness and competitive intensity, respectively, for the $j$ th individual in the $i$ th group, and $z_{i}$ is the average competitive intensity for members of the $i$ th group. Each individual gains a share $z_{i j} / z_{i}$ of the local resources equivalent to its competitive intensity, $z_{i j}$, relative to the average competitive intensity of the group, $z_{i}$. Higher levels of competition reduce the average group productivity, $1-z_{i}$.

The equilibrium for the model in Equation (1) can be found by the standard evolutionarily stable strategy (ESS) method of analysing the derivative $\mathrm{d} w_{i j} / \mathrm{d} z_{i j}=0$ at $z_{i j}=z_{i}=z^{*}$. A difficulty with this method arises when there are interactions between kin, because there are terms that include the slope of group genotype on individual genotype, $\mathrm{d} z_{i} / \mathrm{d} z_{i j}$ (see below). A new method shows that the standard ESS analysis can be used with kin interactions by replacing $\mathrm{d} z_{i} / \mathrm{d} z_{i j}$ with $r$, the kin selection coefficient of relatedness (Frank 1995; Taylor \& Frank 1996). This method is appropriate because the slope of group genotype on individual genotype is the definition of the kin selection coefficient, $r$, in this type of model. Thus the method is simply the standard ESS technique (Maynard Smith 1982) with special extensions to handle kin selection (Taylor \& Frank 1996). The slope $\mathrm{d} z_{i} / \mathrm{d} z_{i j}$ arises from the standard chain rule of basic calculus:

$$
\frac{\mathrm{d} w_{i j}}{\mathrm{~d} z_{i j}}=\frac{\partial w_{i j}}{\partial z_{i j}}+\frac{\partial w_{i j}}{\partial z_{i}} \frac{\mathrm{d} z_{i}}{\mathrm{~d} z_{i j}}=\frac{\partial w_{i j}}{\partial z_{i j}}+r \frac{\partial w_{i j}}{\partial z_{i}}
$$

Solving by this extended ESS method yields the equilibrium $z^{*}=1-r$. Self-restraint evolves when relatedness is high, reducing competition between group members and increasing group success. By contrast, low relatedness leads to intense competition and low group productivity.

If competition within the group can be repressed, then the success of each group member would be increased. Reduced competition would be particularly valuable when relatedness and self-restraint are low. But how can traits that reduce competition evolve when individuals gain by struggling for a larger share of the local resources?

Consider an extension of the previous kin selection model. A second trait, $a$, determines each individual's contribution to a mechanism that reduces competition among all members of the local group (mutual policing). The extended model is

$$
w_{i j}=\left(a_{i}-c a_{i j}+\left(1-a_{i}\right) z_{i j} / z_{i}\right)\left[1-\left(1-a_{i}\right) z_{i}\right],
$$

where $a_{i j}$ is an individual's contribution to mutual policing, and $a_{i}$ is the average level of policing in the local group. The first set of terms has three components which, together, are proportional to an individual's relative fitness within its group. A fraction of interactions, $a_{i}$, are free from competition because they are under control of the policing trait and follow fair distribution of resources. These interactions contribute a fitness value of 1 within the group. The remaining fraction of interactions, $1-a_{i}$, is subject to competition between group members. An individual's success in the group for competitive interactions is $z_{i j} / z_{i}$, so that the total contribution from unpoliced, competitive interactions is $\left(1-a_{i}\right) z_{i j} / z_{i}$. Each individual's success in the group is reduced by the cost of its contribution to policing, $c a_{i j}$.

The second set of terms, $1-\left(1-a_{i}\right) z_{i}$, is the local group's success, of which each individual receives its share according to the first set of terms described above. The assumption here is that 
damage to neighbours caused by the expressed level of competitiveness, $\left(1-a_{i}\right) z_{i}$, reduces group fitness. The expressed level of competitiveness is the intrinsic competitiveness of group members, $z_{i}$, that remain uncontrolled by policing traits, $1-a_{i}$. Expressed competitive traits can damage group success in two different ways. First, neighbours may be harmed directly by destruction of their offspring or personal resources. Second, neighbours may bear an indirect reduction in fitness through the damage that competitive traits cause to the local environment, reducing the sustainable yield of the group. The first case includes direct destruction of a neighbour's eggs; the second includes overgrazing renewable resources so that total productivity is below what could be achieved by prudent exploitation.

\section{Low Relatedness Favours Policing}

A full analysis of evolutionary trends for equation 2 is presented in Frank (1995). Here I explain the key result by focusing on a population in which no policing occurs, and a rare mutant arises that invests some resources in policing.

The equilibrium level of competitiveness is $z^{*}=1-r$ when there is no policing, $a=0$. At this equilibrium the fitness of each individual is $w^{*}=r$. Under what conditions does a rare, policing individual have higher fitness? Suppose the rare mutant invests $a_{i j}=\delta$ in policing, and has the normal value of $z=1-r$ for competitiveness. In the mutant's group, the average level of policing is $a_{i}=r a_{i j}=r \delta$, because the mutant's relatives will tend to share the mutant allele at a rate equal to the kin selection coefficient of relatedness. As mentioned above, the slope of group genotype on individual genotype is the definition of relatedness in group-structured kin selection models. With these values of $a_{i j}$ and $a_{i}$, the fitness of the mutant is

$$
w_{\delta}=(1-c \delta)[1-(1-r \delta)(1-r)] .
$$

The condition for a mutant to increase is $w_{\delta}>w^{*}$, which yields

$$
r<\frac{1-c-c \delta}{1-c \delta},
$$

and, for mutants of small effect (small $\delta$ ),

$$
r<1-c .
$$

Thus low relatedness is more conducive to the spread of a policing trait than is high relatedness
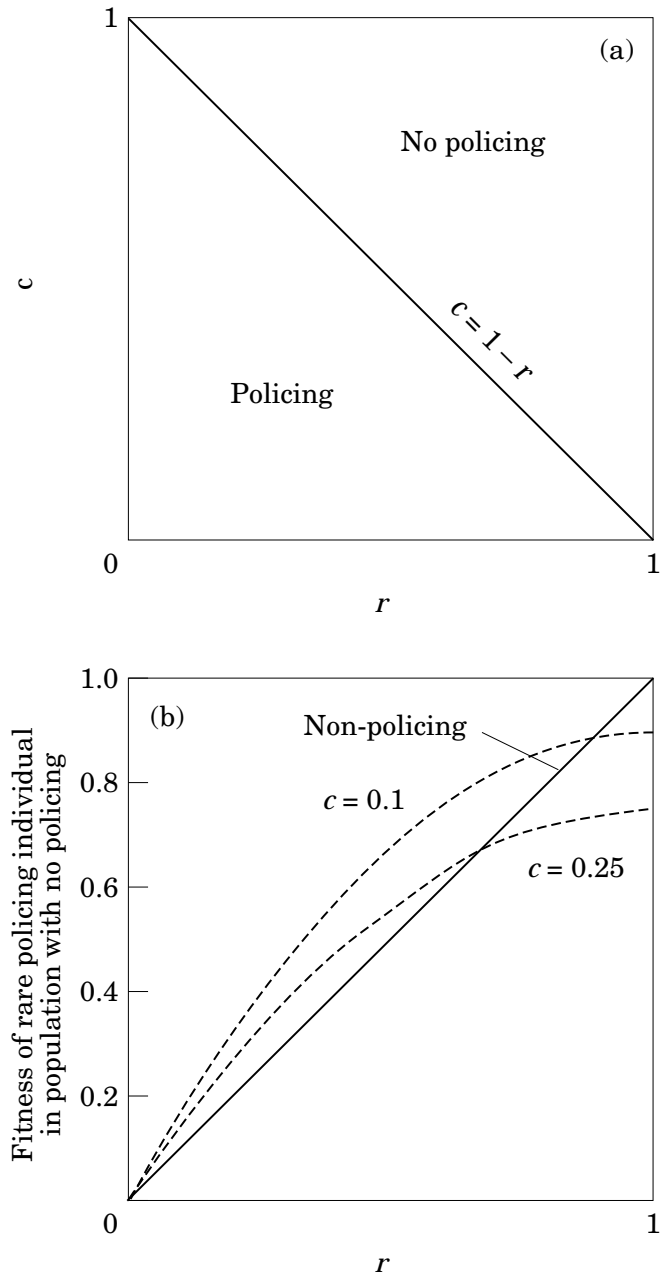

Figure 1. Invasion of policing trait into non-policing population. (a) Low relatedness is more conducive to the increase of policing traits. The condition for the increase of policing is $r<1-c$ when mutations have small effect (small $\delta$ ). (b) Fitness of non-policing (solid line) and policing (dashed lines) individuals in a non-policing population. The dashed lines assume $\delta=1$ and use equation (3) to calculate fitness.

(Fig. 1a). The reason is shown in Fig. 1b. Without policing, fitness follows a line with slope $=1$ because self-restraint causes fitness to equal $r$, the coefficient of relatedness between group members. A rare individual that polices in a group with no policing has fitness $w_{\delta}$. For very low $r$, the policing strategy typically has higher individual fitness than non-policing. The policing curve crosses below the non-policing line at $w^{*}=w_{\delta}$, such that, for high $r$, 
non-policing is favoured. As expected, the lower the cost $c$, the higher is fitness and the higher the value of $r$ at which the curves cross. Thus highcost policing can only be favoured when $r$ is low.

The reason that high-cost policing is favoured only with low $r$ is that the baseline fitness from which a non-policing population starts is $r$. When $r$ is low, group competition is severe and group fitness is low. Any small boost in individual fitness caused by policing is sufficient to provide an advantage. When $r$ is high, individual fitness is already high because self-restraint reduces the group competition. In this case costly policing is likely to lower individual fitness.

\section{Relatedness}

The coefficient of relatedness in the groupstructured models here is the slope of group genotype on individual genotype. For example, with small variations in the policing character, relatedness is given by the derivative

$$
r=\mathrm{d} a_{i} / \mathrm{d} a_{i j},
$$

when evaluated near the population average value of $a$ (Frank 1995; Taylor \& Frank 1996).

If individuals settle randomly into groups, so that pairs of neighbours are unrelated, then $r=1 /$ $N$, where $N$ is the size of the local group. The factor of $1 / N$ arises between unrelated individuals because each individual contributes $1 / N$ to the group average. Put another way, there is a contribution $1 / N$ to $r$ caused by individual selection (Hamilton 1975; Nunney 1985), or, by Wilson's (1980) definitions, the $1 / N$ component of individual selection causes weak altruism in groups. Thus we could write $r=1 / N+r^{\prime}$, where $r^{\prime}$ is the relatedness between pairs of individuals. With random interactions, $r^{\prime}=0$ and $r=1 / N$. In a groupstructured model, where group size $N$ is small relative to the size of the total population, it does not make any sense to consider $r=0$.

I emphasize this point about $r$ because people often assume that random interactions imply $r=0$, and may, therefore, assume that the above models require special group structuring with interactions among kin. The models are, however, formulated in terms of the overall group value of $r$. This formulation is indifferent to the split between benefits returned directly to the actor, at rate $1 / N$, and to neighbours, at a rate equal to the relatedness $r^{\prime}$.
A similar confusion sometimes arises about ratios such as the competitiveness ratio $z_{i j} / z_{i}$ in equation (2). At first glance it appears that, in a population where competitiveness is very low, the denominator $z_{i}$ would be near zero, and an individual could obtain nearly infinite fitness by raising its value of $z_{i j}$. However, this ratio is the individual's competitiveness relative to the average competitiveness of its local group. The individual value $z_{i j}$ is correlated with the group value because the group contains the individual (at a fraction $1 / N$ ) and contains relatives (at a rate $r^{\prime}$ ), so that the overall association between numerator and denominator is determined by $r$. Thus the maximum value of $z_{i j} / z_{i}$ is $1 / r$, and the minimum value of $r$ is $1 / N$. The ratio $z_{i j} / z_{i}$ is therefore bounded above by $N$.

\section{COST OF COMPETITIVENESS}

In the previous section, I showed that, for mutations of small effect, policing invades a nonpolicing population when $r<1-c$. If that condition on relatedness is satisfied, subsequent selection favours policing to increase to $a \rightarrow 1$ (Frank 1995). As policing rises, an increasing proportion of an individual's competitiveness is repressed; one can think of a proportion $a$ of investment in competitiveness that must go to avoiding the police. This repression causes an increasing allocation by individuals to competitive traits, so that as $a \rightarrow 1$, competitiveness increases to $z \rightarrow(1-r) /[r(1-c)]$.

The competitiveness trait, $z$, will often increase above 1 as $a$ increases. The high level of allocation to competitiveness in a policing population, if introduced into a non-policing population, would cause the fitness contribution of these traits to be negative (with $a=0$, average fitness is $1-z$, which is negative when $z>1$ ). This type of incompatibility sometimes raises questions about a model. But the high competitiveness in a policing situation is no different from high internal pressure in a fish that lives at great depth. The fish brought to the surface explodes; intense competition and avoidance of repressive policing cause chaos when the same amount of energy is devoted to competition in the absence of repressive policing.

The model of equation (2) does lack realism, however, because the competitive trait is allowed to increase to any level without accounting for the 

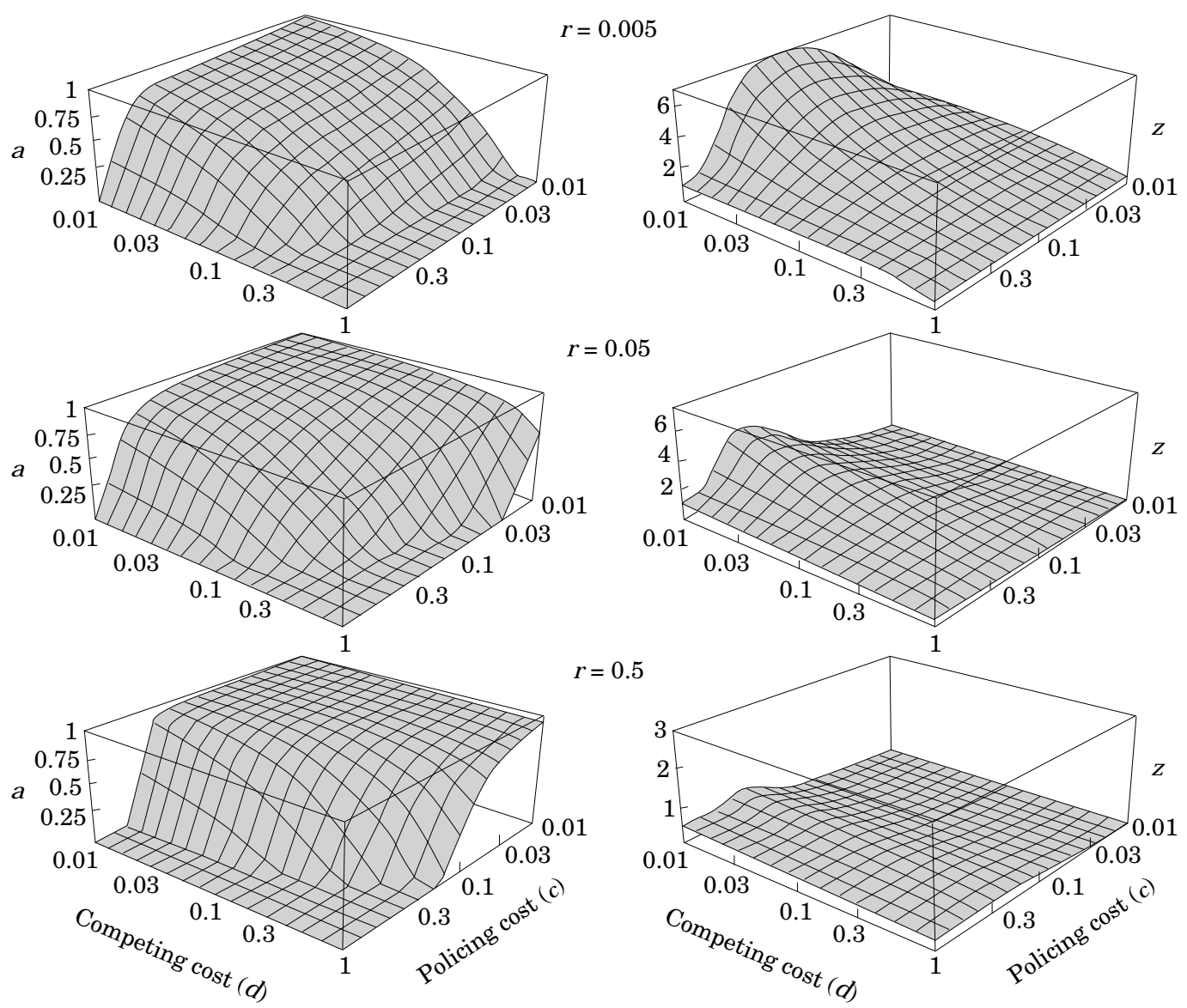

Figure 2. Equilibrium values of policing $(a)$ and competitiveness (z). Results were obtained from equation (6) by solving $\partial w_{i j} / \partial a_{i j}=\partial w_{i j} / \partial z_{i j}=0$ evaluated at $a_{i j}=a^{*}$ and $z_{i j}=z^{*}$, by the methods described in Frank (1995). The resulting simultaneous equations were solved numerically, followed by a check for local stability.

cost of the investment to the individual. The original model is easily extended

$$
\begin{gathered}
w_{i j}=\left(1-c a_{i j}-d z_{i j}\right)\left[a_{i}+\left(1-a_{i}\right) z_{i j} / z_{i}\right] \\
{\left[1-\left(1-a_{i}\right) z_{i}\right],}
\end{gathered}
$$

where $d$ is the cost to the individual of allocation to competitive traits. When $d=0$, equation (6) is slightly different from equation (2), but has the same qualitative and quantitative consequences for the evolution of policing and competitiveness. Here the personal cost terms, $c a_{i j}$ and $d z_{i j}$, are grouped together into a separate fitness component.

When $d>0$, competitiveness is held to a lower level. The equilibrium values of policing $(a)$ and competitiveness $(z)$ are shown in Fig. 2. Inter- mediate values of $a$ and $z$ are common. When $a$ increases from zero, $z$ often increases because part of the competitive allocation is absorbed by repressive policing. When policing rises to a high level, then most of the allocation to competitiveness is wasted because its benefits are repressed. Thus as $a$ increases to high values, $z$ tends to decline.

\section{VARIATION IN RESOURCES}

Individuals often vary in vigour and in the total resources they have available to invest in traits. It seems likely that individual differences in vigour would cause variation in investment to policing and competitiveness. 
The model in equation (6) can be extended to

$$
\begin{array}{r}
w_{i j}=\left(k_{i j}-c a_{i j}-d z_{i j}\right)\left[a_{i}+\left(1-a_{i}\right) z_{i j} / z_{i}\right] \\
{\left[1-\left(1-a_{i}\right) z_{i}\right],}
\end{array}
$$

where the only difference is that the leading one has been replaced by individual resource level, $k_{i j}$. I assume that individual variation in resources is an environmental effect uncorrelated with genotype, and that each individual can adjust its policing and competitive allocation based on its own resource level.

If the resource distribution is the same in every group, we can drop the subscript $i$ and describe the resource distribution with $k_{j}=k+\alpha_{j}$. For simplicity I will assume a symmetric distribution about $k$. (When $k=1$ and $\alpha_{j}=0$ for all $j$, there is no variation in resources, and we have the equilibrium result from the previous section, $a^{*}$ and $z^{*}$.)

When variations are not too large, such that $a^{*}+\alpha_{j} / c \geq 0$ for all $j$, numerical analysis suggests a remarkably simple conclusion. First, variation in resources does not influence investment in competitiveness; everyone invests $z^{*}$. Second, small changes in resource level may cause large changes in the policing trait. At equilibrium, individuals adjust their investment so that everyone has the same individual fitness. Thus relatively strong individuals allocate much to policing, and relatively weak individuals contribute nothing. When $a^{*}$ is small or variation in resources is large, such that $a^{*}+\alpha_{j} / c<0$ for some $j$, the same qualitative conclusions hold. Relatively weak individuals do not contribute to policing and relatively strong individuals do contribute. Most likely, there exists a cut off below which individuals do not contribute, and above which individuals contribute increasingly as their resources increase. I do not, however, have a formal proof of that conjecture.

When the condition $a^{*}+\alpha_{j} / c \geq 0$ is satisfied for all $j$, individual fitness is constant when $k_{j}-c a_{j}^{*}$ is equal to $k-c a^{*}$. Thus, at equilibrium, each individual allocates to policing

$$
a_{j}^{*}=a^{*}+\alpha_{j} / c \text {. }
$$

When the cost of policing, $c$, is small, then small variations in resource level, $\alpha_{j}$, cause large variations in individual behaviour, $a_{j}^{*}$. Figure 3 shows the simplest case, in which there are two resource levels, $k_{1}=k+\alpha_{1}$ and $k_{2}=1+\alpha_{2}$, with $\alpha_{1}=\alpha$ and $\alpha_{2}=-\alpha$. Half of each group has the high resource

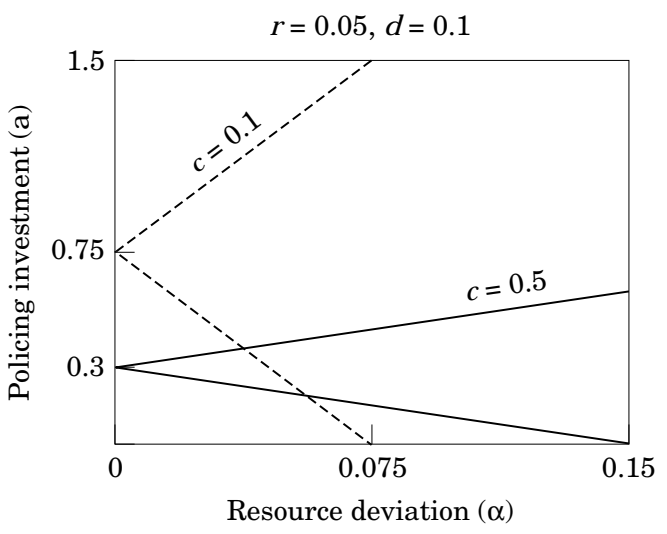

Figure 3. Policing investment when resources vary. For each pair of lines, the upper line shows equilibrium investment in policing for an individual with $k_{j}=1+\alpha$ resources, and the lower line shows investment for an individual with $k_{j}=1-\alpha$ resources. The solution for this model is obtained from equation (7) by solving the $2 \mathrm{~m}$ simultaneous equations $\partial w_{i j} / \partial a_{i j}=\partial w_{i j} / \partial z_{i j}=0$ evaluated at $a_{i j}=a_{j}^{*}$ and $z_{i j}=z_{j}^{*}$ for $j=1, \ldots, m$. The interpretation of relatedness coefficients has not been fully explored in this model, in which behaviour is conditional on environmental effects $k$. These details about relatedness do not, however, affect the main conclusion about divergence of behaviour among individuals with varying resource levels.

level, and the other half has the low level. With a cost of policing of $c=0.1$, resource deviations of $7.5 \%$ cause the high resource group to double its investment over the group average, and the low resource group to allocate no resources to policing.

These results show that, when policing is favoured, small resource variations cause the well endowed to take over social control. This is similar to Clutton-Brock \& Parker's (1995) conclusion that dominant individuals will punish weaker individuals who disrupt group cohesion. However, my models account explicitly for the role of kin selection and require minimal assumptions about memory and strategy. This minimalist approach highlights key processes in the evolution of group cohesion.

\section{ACKNOWLEDGMENTS}

This research is supported by NSF grant DEB9057331 and NIH grant GM42403. 


\section{REFERENCES}

Alexander, R. D. 1987. The Biology of Moral Systems. New York: Aldine de Gruyter.

Axelrod, R. 1984. The Evolution of Cooperation. New York: Basic Books.

Clutton-Brock, T. H. \& Parker, G. A. 1995. Punishment in animal societies. Nature, Lond., 373, 209-216.

Frank, S. A. 1995. Mutual policing and repression of competition in the evolution of cooperation groups. Nature, Lond., 377, 520-522.

Hamilton, W. D. 1964. The genetical evolution of social behaviour. I,II. J. theor. Biol., 7, 1-16, 17-52.

Hamilton, W. D. 1975. Innate social aptitudes of man: an approach from evolutionary genetics. In: Biosocial Anthropology (Ed. by R. Fox), pp. 133-155. New York: Wiley.
Maynard Smith, J. 1982. Evolution and the Theory of Games. Cambridge: Cambridge University Press.

Maynard Smith, J. \& Szathmáry, E. 1995. The Major Transitions in Evolution. San Francisco: Freeman.

Nunney, L. 1985. Female-biased sex ratios: individual or group selection? Evolution, 39, 349-361.

Ratnieks, F. L. W. \& Visscher, P. K. 1989. Worker policing in the honeybee. Nature, Lond., 342, 796797.

Taylor, P. D. \& Frank, S. A. 1996. How to make a kin selection model. J. theor. Biol., 180, 27-37.

Wilson, D. S. 1980. The Natural Selection of Populations and Communities. Menlo Park, California: Benjamin/ Cummings.

Wilson, D. S. \& Sober, E. 1994. Reintroducing group selection into the human behavioral sciences. Behav. Brain Sci., 17, 585-608. 